\title{
Biosensors: Monitoring Human Health for the SDGs Sustainable Development Goals
}

\author{
Hideaki Nakamura* \\ Department of Liberal Arts, Tokyo University of Technology, Japan
}

Received: 制: September 11, 2018; Published: 制 September 20, 2018

*Corresponding author: Hideaki Nakamura, Department of Liberal Arts, Tokyo University of Technology, Hachioji, Tokyo, Japan

\begin{abstract}
Due to global warming, our life is gradually getting worse. There are various problems such as lack of food and depletion of living environment. On the other hand, especially in the developed countries, people with excessive calorie intake and lack of exercise become lifestyle disease. Under these circumstances, it will be difficult for us to live healthily. To improve this situation, the Sustainable Development Goals (SDGs) were set by the United Nations. This mini review focuses on biosensor as a tool to monitor the human health conditions towards achieving the SDGs.
\end{abstract}

\section{Introduction}

A biosensor was developed by the fusion of biology and electronics, and constructed by a biorecognition element and a transducer, it has been widely used as a simple, analyte specific, rapid, low-cost, and energy saving measuring device in the field of the clinical, food and environmental analyses [1]. The first biosensor was developed employing glucose oxidase and a dissolved oxygen electrode for blood glucose monitoring by Updike and Hicks in 1967 [2]. Since, the development of the biosensors has been widespread worldwide and some of them have been commercially available [3]. On the other hand, the United Nations adopted "Transforming our World: the 2030 Agenda for Sustainable Development (2030 Agenda)" and set the Sustainable Development Goals (SDGs) consisting of 17 global goals in 2015 [4]. The 1st goal and the 2nd goal of the SDGs are "No Poverty" and "Zero Hunger", respectively. In fact, future food and nutritional security has become a major concern for both rich and poor, given the present concurrence of rising human population, climate change and changing consumption habits [5]. The nutrition of carbohydrates, proteins, fats, vitamins, minerals, and fiber is required to maintain healthy life. However, it is known that people's nutrition sources are more likely to bias to carbohydrates in countries and regions where food is scarce, or even in developed countries, the situation will be similar depending on living standards. In such cases, people become susceptible to diabetes. On the contrary, people with excessive calorie intake and lack of exercise naturally become susceptible to diabetes.

In the report by World Health Organization (WHO), the number of people with diabetes has risen from 108 million in 1980 to 422 million in 2014 [6]. WHO projects that diabetes will be the seventh leading cause of death in 2030 [6,7]. However, in the 3rd goal of the
SDGs, "Good Health and Well-Being for People" is set. To realize the 3rd goal, such forecast of the diabetes population by WHO needs to be avoided by our efforts.

As one of its efforts, the biosensor to monitor blood glucose level can be expected. Various types of blood glucose biosensors have been developed for practical use to date. There is a selfmonitoring blood glucose (SMBG) biosensor as the most popular one. Early SMBG biosensor (although it is still used today) is finger-prick type and consists of a portable measuring device and a disposable biosensor chip [1]. For the chip design, we had many ideas to reduce manufacturing cost, and sample volume, etc. (over 30 patents applied to PCT) and developed several types of the disposable biosensor chip, for examples, low costs and precise sensor manufacturing [8], a minimally invasive sensor chip $[9,10]$, and a package-free transparent biosensor chip [11,12]. As subsequent development of the SMBG biosensor, we developed several types of needle integrated biosensors $[13,14]$, then we also developed a self-sterilizing lancet to integrate into the biosensor chip [15].

After that, to avoid blood sampling, the SMBG biosensor was improved for continuous monitoring [16]. Currently, the SMBG biosensors are being developed for the next step. Research group of Wang et al. (USA) and Slaughter et al. (USA) are studying on the development of wearable non-invasive epidermal glucose sensors, respectively $[17,18]$, Mitsubayashi et al. are studying on the development of wireless module to apply to a biosniffer (biochemical gas sensor for breath acetone) [19], and Cosnier et al. (France) are studying on the development of implantable self-powering biosensors, which employ enzymatic biofuel cell 
techniques using blood glucose as an energy source $[17,20]$. In the SMBG management, urine glucose biosensors are also play an important role [21]. To achieve the 3rd goal of the SDGs, clinical analysis as one of the medical technologies involved in other diseases is very important, and the biosensors are also expected as one of powerful tools for clinical analysis [4].

By WHO estimation, cancer is the second leading cause of death globally, and was responsible for 8.8 million deaths in 2015 . Globally, nearly 1 in 6 deaths is due to cancer [22]. For the cancer detection using the biosensor, nucleic acid and protein biomarkers have widely been measured as analytes [23]. These biosensors are usually employed nucleic acid or antibody which is specific to the analyte molecule. As a recognition element of nucleic acid, aptamer, DNase, or RNase is employed [24]. Recently, molecularly-imprinted polymer (MIP) is beginning to be used instead of the antibody [25]. Since the concentration of glucose is high around the cancer tissue, the development of a biosensor for surgical operation is also under way [26]. The 3rd goal of the SDGs also includes tackling the problem of HIV/AIDS and malaria [4]. Commercially averrable biosensor checks for antibodies to HIV in an oral fluid sample [3]. As current biosensor method for HIV, approach using sequencespecific DNA or sequence-specific MIP is in progress [27,28]. For malaria as a pathogen, the studies on biosensor is following similar approaches which are described above.

In general, people wish to live healthy life. By WHO estimation, the healthy life expectancy at birth was 63.3 years globally in 2016 [29]. Point of Care Testing (POCT), which allows easy examination and diagnosis due to an increase in lifestyle diseases and an increase in health consciousness, has received much attention especially in developed countries. Recently, from such a background, studies on the biosensors related to POCT have been actively conducted using mobile devices such as smartphone [30-32]. In addition, microflow systems so called "Lab-on a chip" have also been studied very actively [33,34]. Perhaps in 2030, many people may have various biosensors for POCT to keep the healthy life. For healthy life, we need to pay attention to eating habits. Recently, attention has been focused on the antioxidant capacity contained in foods, and we have succeeded in measuring the antioxidant capacity of ingredients contained in vegetables and green tea using our own electrochemical method $[35,36]$.

In order to maintain people's health, it is also important to ensure the quality of the water source that stores drinking water. This is related to both the 6th goal "Clean Water and Sanitation" and the 14th goal "Life Below Water" of the SDGs. However, nutrient salt released from the sewage treatment plant makes it difficult to maintain water quality of the water source $[37,38]$. In order to deal with these problems, we have also developed the biosensors to measure phosphorus with high sensitivity $[39,40]$. Organic pollution is occurred after eutrophication by growth of phytoplankton [41]. Then, we have developed biosensors for fresh water [42] and see water [43]. For water toxicity monitoring, we have developed biosensing methods using living yeast cells which are eukaryote as well as human. One was especially for heavy metal ion toxicity sensing [44], and the other were new concept toxicity assay utilizing damped glycolytic oscillation $[45,46]$.

The oscillation waveform was sensitively and complicatedly changed to various toxicities. We consider that these changes were a consequence of many stimulates based on the various metabolic pathways in the living cell, because the glycolysis is an important system that plays the central role of metabolism. It is also important to maintain healthy agricultural soil with good microflora for plants, as the goal is set by the 15th goal "Life on Land" of the SDGs. However, as it is seen in the Middle East etc., migration and refugees occur due to the devastation of agricultural land, and global concerns are expanding. In such circumstances, we succeeded in developing a microbial biosensor capable of diagnosing the condition of agricultural soil [47], and has been put into practical use for the first time in the world as "Soil Dock ${ }^{\mathrm{TM}}$ " [48].

\section{Conclusion}

In this mini-review, I introduced the biosensors as a tool to monitor human health conditions and the possibilities as a health monitor to achieve the SDGs. Therefore, not only the biosensors for medical use, I also introduced the biosensors for environmental use that are relevant to the SDGs. The details about the microbial biosensors to achieve the SDGs are currently under preparation for publication. When we arrived in 2030, I am looking forward to how biosensors contribute to people's health. Perhaps, it may be a presence that cannot be separated from the hand or the body like the current smartphone.

\section{References}

1. Nakamura H, Karube I (2003) Current research activity in biosensors. Anal Bioanal Chem 377(3): 446-468.

2. Updike SJ, Hicks GP (1967) The enzyme electrode. Nature 214(5092): 986-988.

3. Bahadır EB, Sezginturk MK (2015) Applications of commercial biosensors in clinical, food, environmental, and biothreat/biowarfare analyses. Anal Bio chem 478: 107-120.

4. https://www.un.org/sustainabledevelopment/

5. Porter JR, Xie L, Challinor A, Cochrane K, Howden M, et al. (2014) Food security and food production systems. In Fifth assessment report of the intergovernmental panel on climate change 485-533.

6. http://www.who.int/news-room/fact-sheets/detail/diabetes

7. Mathers CD, Loncar D (2006) Projections of global mortality and burden of disease from 2002 to 2030. PLoS Med 3(11): e442.

8. Nakamura H, Gotoh M, Ishikawa T, Karube I, Hosoya T, et al. (2009) Sensor Chip and Sensor Chip Production Method. US Patent App. 12/083,694.

9. Karube I, Gotoh M, Nakamura H (2007) Biosensor and production method therefor. US Patent App. 10/565,830.

10. Kaimori S, Kitamura T, Ichino M, Hosoya T, Kurusu F, et al. (2006) Structural development of a minimally invasive sensor chip for blood glucose monitoring. Anal Chim Acta 573-574: 104-109.

11. Nakamura H, Shinohara S, Gotoh M, Karube I (2003) Biosensors. US Patent App. 11/087,015

12. Nakamura H, Tohyama K, Tanaka M, Shinohara S, Tokunaga Y et al. (2007) Development of a package-free transparent disposable biosensor chip for simultaneous measurements of blood constituents and investigation of its storage stability. Biosens Bioelectron 23(5): 621-626. 
13. Nakamura H, Gotoh M, Karube I (2009) Needle integrated biosensor. US Patent App. 11/994,010.

14. Ichino M, Hosoya T, Kuwa K, Matsumura N, Nakamura H (2007) Device for measuring constituents in the blood. US Patent D548,347.

15. Nakamura H, Tanaka M, Shinohara S, Gotoh M, Karube I (2007) Development of a self-sterilizing lancet coated with a titanium dioxide photocatalytic nano-layer for self-monitoring of blood glucose. Biosens Bioelectron 22(9-10): 1920-1925.

16. http://www.cygnusaero.com/index.html

17. Kim J, Campbell AS, Wang J (2018) Wearable non-invasive epidermal glucose sensors: A review. Talanta 177: 163-170.

18. Kulkarni T, Mburu N, Slaughter G (2016) Characterization of a selfpowered glucose monitor. Sens Transduce 203(8): 1-7.

19. Chien PJ, Suzuki T, Masato Tsuji M, Ye M, et al. (2017) Biochemical gas sensors (Bio sniffers) using forward and reverse reactions of secondary alcohol dehydrogenase for breath isopropanol and acetone as potential volatile biomarkers of diabetes mellitus. Anal Chem 89(22): 1226112268.

20. Cosnier S, Gross AJ, Giroud F, Holzinger M (2018) Beyond the hype surrounding biofuel cells: What's the future of enzymatic fuel cells? Curr Opini in Electrochem.

21. Nakamura H, Tsuboi Y, Gotoh M (2012) A simple potentiometric urine glucose biosensor using a paper-based disposable reagent sheet and a mobile pH meter. Curr Topics in Anal Chem 9: 71-75.

22. http://www.who.int/news-room/fact-sheets/detail/cancer

23. Jayanthi VSA, Das AB, Saxena U (2017) Recent advances in biosensor development for the detection of cancer biomarkers. Biosens Bioelectron 91: 15-23.

24. Chen A, Yang S (2015) Replacing antibodies with aptamers in lateral flow immunoassay. Biosens Bioelectron 71: 230-242.

25. Uzun L, Turner APF (2016) Molecularly-imprinted polymer sensors: Realising their potential. Biosens Bioelectron 76: 131-144.

26. Nakamura H, Mogi Y, Akimoto T, Naemura K, Kato T, et al. (2008) An enzyme-chromogenic surface plasmon resonance biosensor probe for hydrogen peroxide determination using a modified Trinder's reagent. Biosens Bioelectron 24(3): 455-460.

27. Herzner AM, Hagmann CA, Goldeck M, Wolter S, Kubler K (2015) Sequence-specific activation of the DNA sensor cGAS by Y-form DNA structures as found in primary HIV-1 cDNA. Nature Immunol 16: 10251033.

28. Babamiri B, Salimi A, Hallaj R (2018) A molecularly imprinted electrochemiluminescence sensor for ultrasensitive HIV-1 gene detection using EuS nanocrystals as luminophore. Biosens Bioelectron 117: 332-339.

29. http://www.who.int/gho/mortality_burden_disease/life_tables/hale/ en/

30. Turner APF (2017) Enabling mobile health. Procedia Technol 27: 4-5.

31. Zhang D, Liu Q (2016) Biosensors and bioelectronics on smartphone for portable biochemical detection. Biosens Bioelectron 75: 273-284.

32. Xu D, Huang X, Guo J, Ma X (2018) Automatic smartphone-based microfluidic biosensor system at the point of care. Biosens Bioelectron 110: 78-88
33. Nakamura H, Murakami Y, Yokoyama K, Tamiya E, Suda M, et al. (2001) A compactly integrated flow cell with a chemiluminescent FIA system for determining lactate concentration in serum. Anal Chem 73(2): 373-378.

34. Samiei E, Tabrizian M, Hoorfar M (2016) A review of digital microfluidics as portable platforms for lab-on a-chip applications. Lab on a Chip 16 : 2376-2396.

35. Gotoh M, Hirose H, Ishikawa T, Nakamura H, Yokoyama K (2015) Establishment of ferricyanide chronoamperometric total antioxidant capacity assay employing a carbon screen-printed disposable microchip - Fundamental study using vegetable extraction -. Sens Materials 27(9): 825-838.

36. Nakamura H, Nakayama Y, Gotoh M (2017) Ferricyanide chronoamperometric total antioxidant capacity assay for green tea. Curr Topics in Anal Chem 10: 29-34.

37. Nakamura H (2018) Current status of water environment and their microbial biosensor techniques- Part I: Part I: Current data of water environment and recent studies on water quality investigations in Japan, and new possibility of microbial biosensor techniques. Anal Bioanal Chem 410(17): 3953-3965.

38. Nakamura H (2018) Current status of water environment and their microbial biosensor techniques -Part II: Recent trends in microbial biosensor development. Anal Bioanal Chem 410(17): 3967-3989.

39. Nakamura H, Tanaka H, Hasegawa M, Masuda Y, Arikawa Y, et al. (1999) An automatic flow-injection analysis system for determining phosphate ion in river water using pyruvate oxidase G (from Aerococcus viridans). Talanta 50(4): 799-807.

40. Nakamura H (2001) Phosphate ion determination in water for drinking using biosensors. Bunseki kagaku 50(8): 581-582.

41. Nakamura H (2010) Recent organic pollution and its biosensing methods. Anal Meth 2(5): 430-444.

42. Nakamura H, Hirata Y, Mogi Y, Kobayashi S, Suzuki K, et al. (2007) A simple and highly repeatable colorimetric toxicity assay method using 2, 6-dichlorophenolindophenol as the redox color indicator and whole eukaryote cells. Anal Bioanal Chem 389(3): 835-840.

43. Nakamura H, Mogi Y, Hattori H, Kita Y, Hattori D, et al. (2008) Absorption-based highly sensitive and reproducible biochemical oxygen demand measurement method for seawater using salt-tolerant yeast Saccharomyces cerevisiae ARIF KD-003. Anal Chim Acta 620(1-2): 127133.

44. Nakamura H, Suzuki K, Ishikuro H, Kinoshita S, Koizumi R, et al. (2007) A new BOD estimation method employing a double-mediator system by ferricyanide and menadione using the eukaryote Saccharomyces cerevisiae. Talanta 72(1): 210-216.

45. Nakamura H, Suzuki M (2007) New concept for a toxicity assay based on multiple indexes from the wave shape of damped metabolic oscillation induced in living yeast cells (part I): characterization of the phenomenon. Anal Bioanal Chem 389(4): 1225-1232.

46. Nakamura H, Suzuki M (2007) New concept for a toxicity assay based on multiple indexes from the wave shape of damped metabolic oscillation induced in living yeast cells (part II): application to analytical toxicology. Anal Bioanal Chem 389(4): 1233-1241.

47. Hashimoto Y, Nakamura H, Asaga K, Karube I (2008) A new diagnostic method for soil-borne disease using a microbial biosensor. Microbes Environ 23(1): 35-39.

48. https://www.sakataseed.co.jp/corporate/news/20060530.htmls 


\section{ISSN: 2574-1241}

DOI: $10.26717 / B J S T R .2018 .09 .001761$

Hideaki Nakamura. Biomed J Sci \& Tech Res

(c) (i) This work is licensed under Creative

Submission Link: https://biomedres.us/submit-manuscript.php

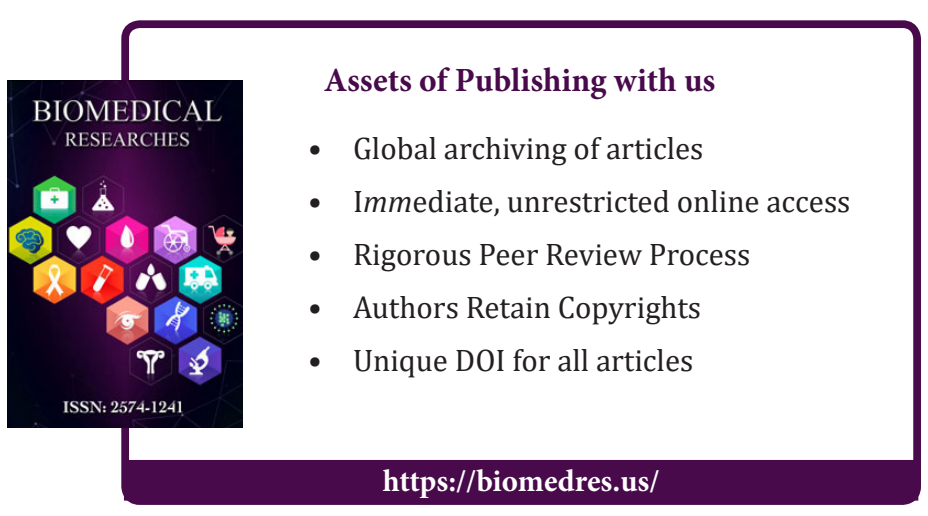

